\title{
Development of a machine vision system for real-time monitoring and control of batch flotation process
}

\begin{abstract}
Substantial progresses have been made over the past decade in using machine vision for automatic control of the froth flotation process. A machine vision system is able to extract the visual features from the captured froth images and present the results to process control systems. The current research work is concerned with the development and implementation of a machine vision system for real time monitoring and control of a batch flotation system. The proposed model-based control system comprises two in-series models connecting the process variables to the froth features and the metallurgical parameters along with a stabilizing fuzzy controller. The results indicate the developed machine vision based control system is able to accurately predict the metallurgical parameters of the existing batch flotation system from the extracted froth features and efficiently maintain them at their setpoints despite step disturbances in the process variables. Furthermore, the proposed control system leads to higher target values for the metallurgical parameters than the previously developed system $(\mathrm{RCu}=91.1 \% ; \mathrm{GCu}=11.2 \%$ vs. $\mathrm{RCu}=87.6 \% ; \mathrm{GCu}=8.1 \%)$.
\end{abstract}

Keyword: Froth flotation; Machine vision; Froth model; Fuzzy controller 